\title{
Pyrotinib plus capecitabine for human epidermal growth factor receptor 2-positive metastatic breast cancer after trastuzumab and taxanes (PHENIX): a randomized, double-blind, placebo- controlled phase 3 study
}

\author{
Min Yan ${ }^{1 \#}$, Li Bian ${ }^{2 \#}$, Xichun $\mathrm{Hu}^{3}$, Qingyuan Zhang ${ }^{4}$, Quchang Ouyang ${ }^{5}$, Jifeng Feng ${ }^{6}$, Yongmei Yin ${ }^{7}$, \\ Tao Sun ${ }^{8}$, Zhongsheng Tong ${ }^{9}$, Xiaojia Wang ${ }^{10}$, Herui Yao ${ }^{11}$, Jianjun Zou ${ }^{12}$, Xiaoyu Zhu ${ }^{12}$, Zefei Jiang ${ }^{2}$ \\ ${ }^{1}$ The Affiliated Cancer Hospital of Zhengzhou University \& Henan Cancer Hospital, Zhengzhou, China; ${ }^{2}$ The Fifth Medical Center of Chinese \\ PLA General Hospital, Beijing, China; ${ }^{3}$ Fudan University Shanghai Cancer Center, Shanghai, China; ${ }^{4}$ Harbin Medical University Cancer Hospital, \\ Harbin, China; ${ }^{5}$ The Affiliated Cancer Hospital of Xiangya School of Medicine, Central South University, Changsha, China; ${ }^{6}$ Jiangsu Cancer \\ Hospital and Jiangsu Institute of Cancer Research and Nanjing Medical University Affiliated Cancer Hospital, Nanjing, China; ${ }^{7}$ The First Affiliated \\ Hospital of Nanjing Medical University, Nanjing, China; ${ }^{8}$ Liaoning Cancer Hospital and Institute, Liaoning, China; ${ }^{9}$ Tianjin Medical University \\ Cancer Institute and Hospital, Tianjin, China; ${ }^{10}$ Zhejiang Cancer Hospital, Hangzhou, China; ${ }^{11}$ Guangdong Provincial Key Laboratory of Malignant \\ Tumor Epigenetics and Gene Regulation, Sun Yat-sen Memorial Hospital, Sun Yat-sen University, Guangzhou, China; ${ }^{12}$ Jiangsu Hengrui Medicine \\ Co. Ltd., Shanghai, China \\ Contributions: (I) Conception and design: Z Jiang, J Zou; (II) Administrative support: Z Jiang, J Zou; (III) Provision of study materials or patients: Z \\ Jiang, M Yan, L Bian, X Hu, Q Zhang, Q Ouyang, J Feng, Y Yin, T Sun, Z Tong, X Wang, H Yao; (IV) Collection and assembly of data: Z Jiang, \\ M Yan, L Bian, X Hu, Q Zhang, Q Ouyang, J Feng, Y Yin, T Sun, Z Tong, X Wang, H Yao; (V) Data analysis and interpretation: All authors; (VI) \\ Manuscript writing: All authors; (VI) Final approval of manuscript: All authors. \\ \#These authors contributed equally to this work. \\ Correspondence to: Prof. Zefei Jiang. Department of Breast Cancer, The Fifth Medical Center of Chinese PLA General Hospital, Beijing 100071, \\ China. Email: jiangzefei@csco.org.cn.
}

Background: Pyrotinib is an irreversible pan-ErbB inhibitor targeting epidermal growth factor receptor, human epidermal growth factor receptor 2 (HER2), and HER4. This randomized, double-blinded phase 3 study evaluated the efficacy and safety of pyrotinib plus capecitabine for HER2-positive local relapsed or metastatic breast cancer.

Methods: Patients who had been treated with trastuzumab and taxanes were randomized $(2: 1)$ to receive either oral pyrotinib or placebo (400 mg, qd) plus capecitabine (1,000 mg/m², bid on days 1-14) for 21-day cycles, using stratified block randomization. The primary endpoint was progression-free survival (PFS) per independent review committee. Patients who progressed on placebo plus capecitabine received subsequent pyrotinib monotherapy. This study is registered with ClinicalTrials.gov (NCT02973737), enrollment is closed.

Results: Between Jul 25, 2016 and Nov 27, 2017, 279 patients were randomly assigned to pyrotinib (n=185) and placebo ( $\mathrm{n}=94)$ groups. As of May 27, 2018, median PFS was 11.1 months [95\% confidence interval (CI), 9.7-16.5] vs. 4.1 months (95\% CI, 2.8-4.2) in the pyrotinib vs. placebo groups, respectively [hazard ratio, 0.18 (95\% CI, 0.13-0.26); $\mathrm{P}<0.001]$. Seventy-one patients in the placebo group subsequently received pyrotinib, showing a response rate of 38.0\% (95\% CI, 26.7-49.3\%) and median PFS of 5.5 months (95\% CI, 4.1-6.9). The most frequent grade 3 or 4 treatment-related adverse events were diarrhea (30.8\% vs. $12.8 \%)$ and handfoot syndrome (15.7\% vs. $5.3 \%)$. No treatment-related deaths were reported.

Conclusions: For HER2-positive local relapsed or metastatic breast cancer after prior trastuzumab and taxanes, pyrotinib plus capecitabine yielded a statistically significant increase in PFS over placebo plus capecitabine. Pyrotinib monotherapy also showed potent anti-tumor activity. 
Keywords: Breast cancer; pyrotinib; human epidermal growth factor receptor 2 (HER2); phase 3

Received: 26 May 2020; Accepted: 28 June 2020; Published: 30 July 2020.

doi: $10.21037 /$ tbcr-20-25

View this article at: http://dx.doi.org/10.21037/tbcr-20-25

\section{Introduction}

Overexpression of human epidermal growth factor receptor 2 (HER2) and/or amplification of HER2 gene occur in approximately $20 \%$ of breast cancers (1). HER2-positive breast cancer tends to be aggressive, resulting in poor prognosis (2-5). With the development of anti-HER2 therapies, great progress has been made in the treatment outcomes of HER2-positive breast cancer. However, therapy landscape is influenced not only by evolving treatment guidelines but also by socioeconomic factors. In the real-world, trastuzumab combined with chemotherapy remains the main choice for early-stage HER2-positive breast cancer and/or initial therapy for metastatic disease.

The actual use of anti-HER2 therapies varies in different countries and regions. According to observational studies conducted between 2000 and 2015 after trastuzumab had been approved for HER2-positive metastatic breast cancer, approximately $12 \%$ of patients in the United States and $27-54 \%$ in Europe did not receive trastuzumab-based regimens or other anti-HER2 agents as first-line and/ or later-line treatment (6-8). Trastuzumab was approved in China in 2002 for HER2-positive metastatic breast cancer. Of the patients in resource-abundant regions (gross domestic product per capita $>\$ 15,000$ and trastuzumab available through Medicare), $87.5 \%$ received trastuzumab for metastatic disease, compared with $42.3 \%$ of the patients in resource-limited regions (9). Lapatinib and pertuzumab were approved in 2013 and 2018, respectively, but its high cost prohibits accessibility, and ado-trastuzumab emtansine (T-DM1) has not yet been approved in China. Drug resistance is also still a major challenge (10-12). Thus, the development of alternative anti-HER2 agents is required.

Pyrotinib is a small-molecule, irreversible pan-ErbB receptor tyrosine kinase inhibitor (TKI) targeting epidermal growth factor receptor, HER2, and HER4 (13). Phase 1 studies demonstrated that pyrotinib monotherapy or in combination with capecitabine was well-tolerated in pretreated HER2-positive metastatic breast cancer (14-16). We designed this randomized, double-blinded phase 3 study (PHENIX) to assess the therapeutic strategy with pyrotinib plus capecitabine after trastuzumab. Considering the therapy landscape in the real-world, placebo plus capecitabine was used in the control arm. Based on scientific and ethical considerations, patients in the control arm could be given pyrotinib monotherapy after disease progression. We present the following article in accordance with the CONSORT reporting checklist (available at http://dx.doi. org/10.21037/tbcr-20-25).

\section{Methods}

\section{Study design}

PHENIX was a randomized, double-blinded, placebocontrolled, multicenter phase 3 trial conducted at 22 sites in China (Table S1). Patients were randomly assigned (2:1) to pyrotinib plus capecitabine or placebo plus capecitabine by stratified block randomization with a block size of six, via an interactive web-based response system with a dynamic randomization list. Stratification factors included presence of visceral disease (yes vs. no) and hormone receptor status [estrogen receptor (ER)- and/or progesterone receptor (PR)-positive $v s$. ER- and PR-negative]. The randomization sequence was generated by the sponsor's randomization specialist. The investigators registered patients at each study centre via the web-response system and assigned them on the basis of the randomization sequence directly obtained from the system. The web-response system ensured that the container sequence was concealed.

The sponsor, investigators, site staff, and patients were masked to treatment assignment. An independent radiologic committee was used with an independent third-party central radiology contractor (Fantastic Bioimaging, TigerMed, Hangzhou, China). Imaging data were evaluated by third-party radiologists using a blinded two reader batch-mode paradigm. Any discrepancies between their evaluations were adjudicated by a third, similarly blinded, independent radiologist. The independent data monitoring committee (IDMC) reviewed the unblinded data and made recommendations regarding continuation/discontinuation of the study. This trial is registered with ClinicalTrials.gov (NCT02973737). 
The protocol and all amendments were approved by the Ethics Committee of each participating site (Table S2). The study was conducted in accordance with Helsinki Declaration of 1964 (revised 2013), Good Clinical Practice, and Chinese laws and regulatory requirements. All patients provided written informed consent.

\section{Patients}

Eligible participants were aged 18-75 years, had histologically confirmed HER2-positive local relapsed or metastatic breast cancer, had received trastuzumab and taxanes, and had up to two prior lines of chemotherapy for relapsed or metastatic disease. HER2 status was assessed according to the 2013 American Society of Clinical Oncology/College of American Pathologists guidelines (17). Patients should have received prior trastuzumab for at least three months in adjuvant setting or at least two 3 -weekly cycles for relapsed or metastatic disease and were not amenable or available for trastuzumab or lapatinib treatment. Patients with brain metastases that were symptomatic or required therapy to control symptoms were excluded. The full inclusion and exclusion criteria are shown in the Supplemental material.

\section{Treatment}

Patients were given continuous oral pyrotinib or placebo at a dose of $400 \mathrm{mg}$ once daily, both in combination with oral capecitabine at a dose of $1,000 \mathrm{mg} / \mathrm{m}^{2}$ twice daily on days 1-14 of each 21-day cycle until disease progression, unacceptable toxicity, withdrawal of consent, or withdrawal by the investigator. To manage adverse events (AEs), capecitabine administration could be interrupted, and dose reductions to $75 \%$ and $50 \%$ of the initial dose were allowed according to a predefined algorithm. Pyrotinib or placebo could be delayed for up to 2 weeks, and dose reductions to 320 and $240 \mathrm{mg}$ per day were permitted according to a predefined algorithm. Patients who progressed on placebo plus capecitabine were allowed to receive pyrotinib monotherapy (400 $\mathrm{mg}$ once daily, 21 days a cycle) at the investigator's discretion. Upon study unblinding, patients who did not progressed on placebo plus capecitabine were allowed to crossover to pyrotinib plus capecitabine.

\section{Outcomes and assessments}

The primary endpoint was independent review committee
(IRC)-assessed progression-free survival (PFS). Secondary endpoints included investigator-assessed PFS, IRC- and investigator-assessed objective response rate (ORR), disease control rate (DCR), clinical benefit rate (CBR), and duration of response (DoR), overall survival (OS), and safety. A complete or partial response (CR or PR) required confirmation at least 4 weeks after the initial response.

Radiographic examinations were conducted every 2 cycles for the first 20 cycles and every 4 cycles thereafter. Tumour responses were assessed by the investigator and IRC based on RECIST version 1.1.

Safety assessments included 12-lead electrocardiograms, vital signs, laboratory tests, echocardiography, and AEs. Cardiac monitoring with echocardiography was performed every 12 weeks. AEs were monitored continuously until 28 days after the last dose and recorded according to the National Cancer Institute Common Terminology Criteria for Adverse Events (NCI CTCAE; version 4.0).

\section{Statistical analyses}

Efficacy analyses were done in the full analysis set, which included all randomized patients who received at least one dose of study drugs. Safety was assessed in patients who received study drugs. Time-to-event endpoints including PFS, OS, and DoR were estimated with the KaplanMeier method and compared between treatment groups with the log-rank test stratified by the randomization strata; stratified Cox proportional-hazards models were used to estimate hazard ratios (HRs) for progression/ death with $95 \%$ confidence intervals (CIs). To explore the effect of prespecified baseline prognostic factors on PFS, a subgroup analysis using the Cox proportionalhazards model was conducted and results were shown in a forest plot. Proportions with regard to the responses were compared with Fisher's exact test. All statistical analyses were performed using SAS (version 9.2).

Assuming a median PFS of 4.5 months for placebo plus capecitabine and 6.5 months for pyrotinib plus capecitabine, 262 events of disease progression or death were required to provide $80 \%$ power to detect the difference in PFS between groups, as denoted by a HR of 0.69 , using a log-rank test at a one-sided significance level of 0.025 . Considering $10 \%$ of proportion of non-evaluable patients, the original planned sample size was approximately 350 .

Due to differences observed between groups in the number of PFS events, the IDMC suggested an $a d-b o c$ interim analysis immediately with 72 PFS events recorded. 
To keep the overall type I error at the same level as planned, an extremely small alpha was spent as 0.00002 by using the Lan-DeMets (O'Brien-Fleming) alpha spending function. The unblinded results were only reviewed by the IDMC. Interim analysis showed that the median PFS was 11.2 months (95\% CI, 8.3-not reached) in the pyrotinib group vs. 4.2 months (95\% CI, 2.8-4.2) in the placebo group [HR, 0.19 (95\% CI, 0.12-0.31); $\mathrm{P}<0.000001]$. Based on both safety and efficacy results, the IDMC recommended to cease further enrolment, but to continue the study for up to 6 months, in order to ensure maturity of data and in turn, the reliability of the study conclusion. Consequently, further enrolment was stopped and the second interim analysis was conducted after the last patient was followed up for 6 months. The superiority boundary for the second interim analysis was re-calculated to have the nominal onesided $\mathrm{P}$ value of 0.0043 by using Lan-DeMets (O'BrienFleming) alpha spending function. Herein, we reported findings of the second interim analysis, based on which the IDMC reported that the efficacy boundary had been crossed, and recommended early termination of the study without further analysis. The study then was unblinded and the patients in the placebo group crossed over to receive pyrotinib plus capecitabine.

\section{Results}

\section{Patients}

Enrolment began on Jul 25, 2016 and was completed on Nov 27, 2017 according to recommendations from the IDMC. A total of 279 eligible patients were randomly assigned, 185 to receive pyrotinib plus capecitabine (pyrotinib group) and 94 to receive placebo plus capecitabine (placebo group; see Figure 1). Baseline characteristics were generally well balanced between the two groups (Table 1).

At the time of the second interim analysis (May 27, 2018 , i.e., 6 months after last patient recruitment), the median duration of follow-up was 8.6 months (range, 0.9-20.7 months) in the pyrotinib group and 8.9 months (range, 1.4-21.2 months) in the lapatinib group. Totally, 109 patients $(58.9 \%)$ in the pyrotinib group and 89 patients $(94.7 \%)$ in the placebo group discontinued treatment (Figure 1). Of the 82 patients who progressed on placebo plus capecitabine, 71 received open-label pyrotinib. At data cutoff, 46 patients (64.8\%) discontinued pyrotinib. Upon study unblinding, the five patients who had not progressed on placebo plus capecitabine were given pyrotinib plus capecitabine per protocol. Of them, two had been treated for 24 cycles as to Apr 30, 2020 and were still continuing treatment.

\section{Efficacy}

According to the IRC assessment, $162(58.1 \%)$ of the 279 patients had disease progression or died, including $84(45.4 \%)$ of the 185 patients in the pyrotinib group and $78(83.0 \%)$ of the 94 patients in the placebo group. An early separation between the two groups was shown in the Kaplan-Meier curves and continued over time (Figure 2A). The median PFS per IRC was significantly prolonged by 7.0 months, from 4.1 months (95\% CI, 2.8-4.2) in the placebo group to 11.1 months (95\% CI, 9.7-16.5) in the pyrotinib group [HR, 0.18 (95\% CI, 0.13-0.26); one-sided $\mathrm{P}<0.001$ ]. Meanwhile, investigator assessment showed the median PFS to be 10.9 months (95\% CI, 8.3-12.4) in the pyrotinib group, which was significantly longer compared with 4.1 months (95\% CI, 3.5-4.2) in the placebo group [HR, 0.24 (95\% CI, 0.17-0.33); one-sided $\mathrm{P}<0.001$; Figure S1].

The ORR was $68.6 \%$ (95\% CI, $61.4-75.3 \%$ ) vs. $16.0 \%$ (95\% CI, 9.2-25.0\%) in the pyrotinib vs. placebo group $(\mathrm{P}<0.001$; Table 2$)$. Of note, 12 patients $(6.5 \%)$ in the pyrotinib group achieved CR, compared with none in the placebo group. The median DoR was 12.2 months (95\% CI, 9.5-not reached) in the pyrotinib group and 4.2 months (95\% CI, 4.1-8.2) in the placebo group $(\mathrm{P}<0.001)$. The DCR was higher in the pyrotinib group [91.9\% (95\% CI, 87.0-95.4\%) vs. 64.9\% (95\% CI, 54.4-74.5\%); $\mathrm{P}<0.001]$. Similarly, the CBR was increased in the pyrotinib group [76.8\% (95\% CI, 70.0-82.6\%) vs. $22.3 \%$ (95\% CI, 14.4-32.1\%); $\mathrm{P}<0.001]$. Investigator-assessed tumour response showed consistent results (Table S3).

Among the 71 patients who received pyrotinib monotherapy after progression on placebo plus capecitabine, $43(60.6 \%)$ had disease progression or died; investigator-assessed median PFS was 5.5 months (95\% CI, 4.1-6.9; Figure 2B). In total, 27 patients achieved objective responses, including one $\mathrm{CR}$ and $26 \mathrm{PR}$ (Table 2). ORR was $38.0 \%$ (95\% CI, 26.7-49.3\%), DCR was $80.3 \%$ (95\% CI, 71.0-89.5\%), and CBR was $42.3 \%$ (95\% CI, 30.8-53.7\%).

As of data cutoff, there were 41 deaths (14.7\%), including 23 deaths $(12.4 \%)$ in the pyrotinib group and $18(19.1 \%)$ in the placebo group. Median OS in both groups had not been reached.

The PFS benefit of pyrotinib plus capecitabine was 


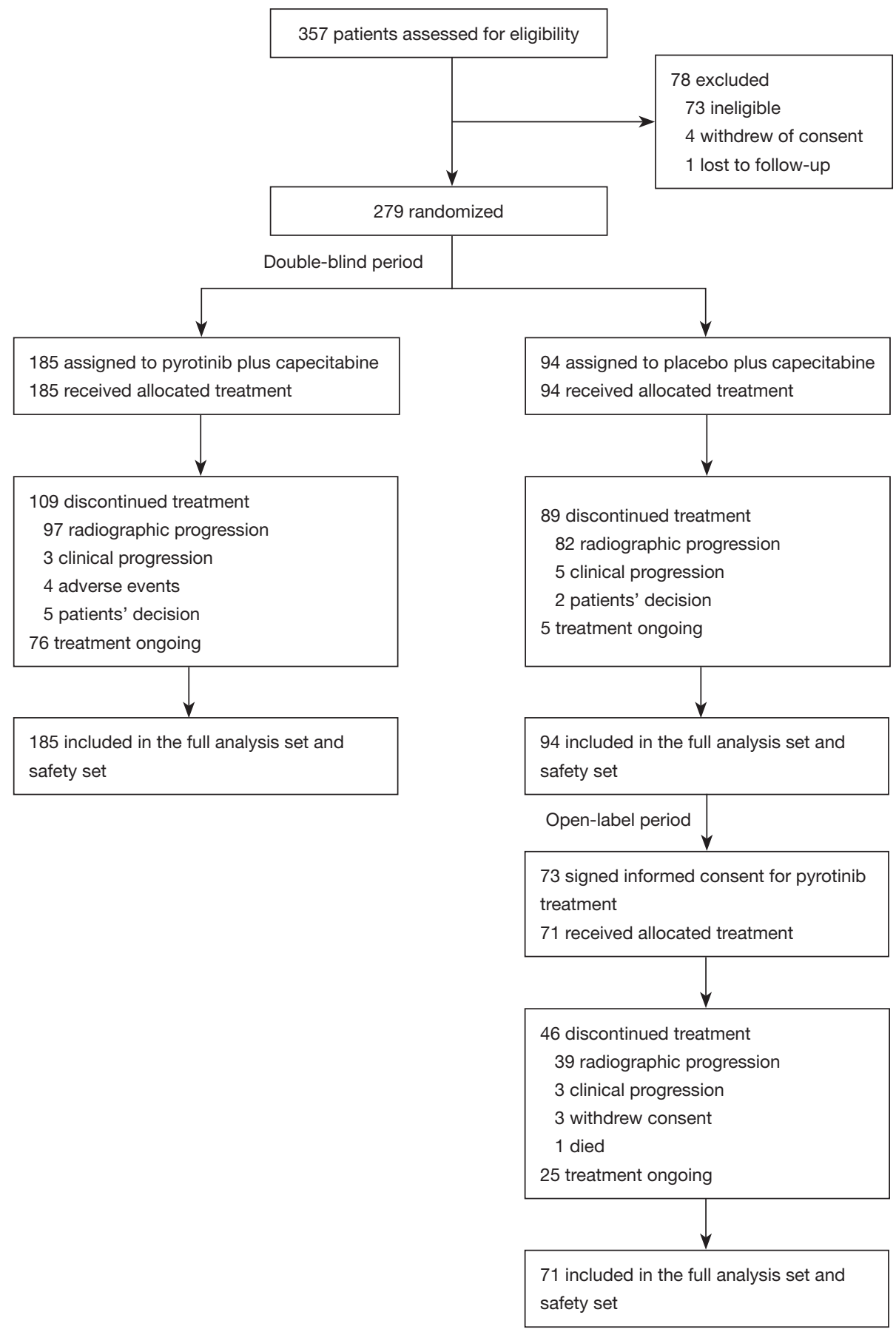

Figure 1 Trial profile.

observed across all predefined subgroups (HRs $<1$, Figure 3). Regardless of treatment line, pyrotinib plus capecitabine showed prolonged PFS [as first-line: 12.5 months (95\% CI, 9.7-not reached) vs. 2.8 months (95\% CI, 2.6-5.6), $\mathrm{P}<0.001$; as second-line: 11.0 months (95\% CI, 8.3-13.6) vs. 4.1 months (95\% CI, 2.8-5.5), $\mathrm{P}<0.001$; as third-line: 9.7 months (95\% CI, 5.5-not reached) vs. 2.8 months (95\% CI, 1.4-4.2), $\mathrm{P}<0.001]$. PFS benefits with pyrotinib plus capecitabine were shown both in those with baseline brain metastases [6.9 months (95\% CI, 5.4-not reached) vs. 
Table 1 Baseline characteristics of patients

\begin{tabular}{|c|c|c|}
\hline Characteristics & Pyrotinib plus capecitabine $(n=185)$ & Placebo plus capecitabine $(n=94)$ \\
\hline \multicolumn{3}{|l|}{ ECOG performance status } \\
\hline 0 & $80(43.2)$ & $30(31.9)$ \\
\hline 1 & $105(56.8)$ & $64(68.1)$ \\
\hline HER2 amplification by FISH & $67(36.2)$ & $40(42.6)$ \\
\hline \multicolumn{3}{|l|}{ Hormone receptor status $^{\dagger}$} \\
\hline ER- and/or PR-positive & $100(54.1)$ & $51(54.3)$ \\
\hline ER- and PR-negative & $85(45.9)$ & $43(45.7)$ \\
\hline Non-visceral & $38(20.5)$ & $22(23.4)$ \\
\hline \multicolumn{3}{|l|}{ Brain metastases at screening } \\
\hline $\mathrm{N}$ & 21 & 10 \\
\hline Received local therapy & $6(28.6)$ & $2(20.0)$ \\
\hline Did not receive local & $15(71.4)$ & $8(80.0)$ \\
\hline \multicolumn{3}{|l|}{ Number of previous therapy lines for advanced disease } \\
\hline 0 & $68(36.8)$ & $27(28.7)$ \\
\hline 1 & $70(37.8)$ & $47(50.0)$ \\
\hline Both & $14(7.6)$ & $9(9.6)$ \\
\hline \multicolumn{3}{|l|}{ Duration of prior trastuzumab therapy for advanced disease ${ }^{\#}$} \\
\hline $\mathrm{N}$ & 98 & 57 \\
\hline Duration, days & $170[2-2,154]$ & 144 [1-701] \\
\hline$<6$ weeks & $13(13.3)$ & $10(17.5)$ \\
\hline $6-12$ weeks & $13(13.3)$ & 11 (19.3) \\
\hline$>12$ weeks & $72(73.5)$ & 36 (63.2) \\
\hline
\end{tabular}

Data are median [range] or $\mathrm{n}(\%)$ unless otherwise indicated. ${ }^{\dagger}$, stratification factor; ${ }^{*}$, percentages were calculated among 175 patients in the pyrotinib group and 86 patients in the placebo group, respectively, whose HER2 status were tested using immunohistochemistry; ${ }^{\#}$, the data of 16 patients in the pyrotinib group and 6 patients in the placebo group were missing. ECOG, Eastern Cooperative Oncology Group; FISH, fluorescence in-situ hybridization; HER2, human epidermal growth factor receptor 2; ER, estrogen receptor; PR, progesterone receptor. 
A Double-blind period

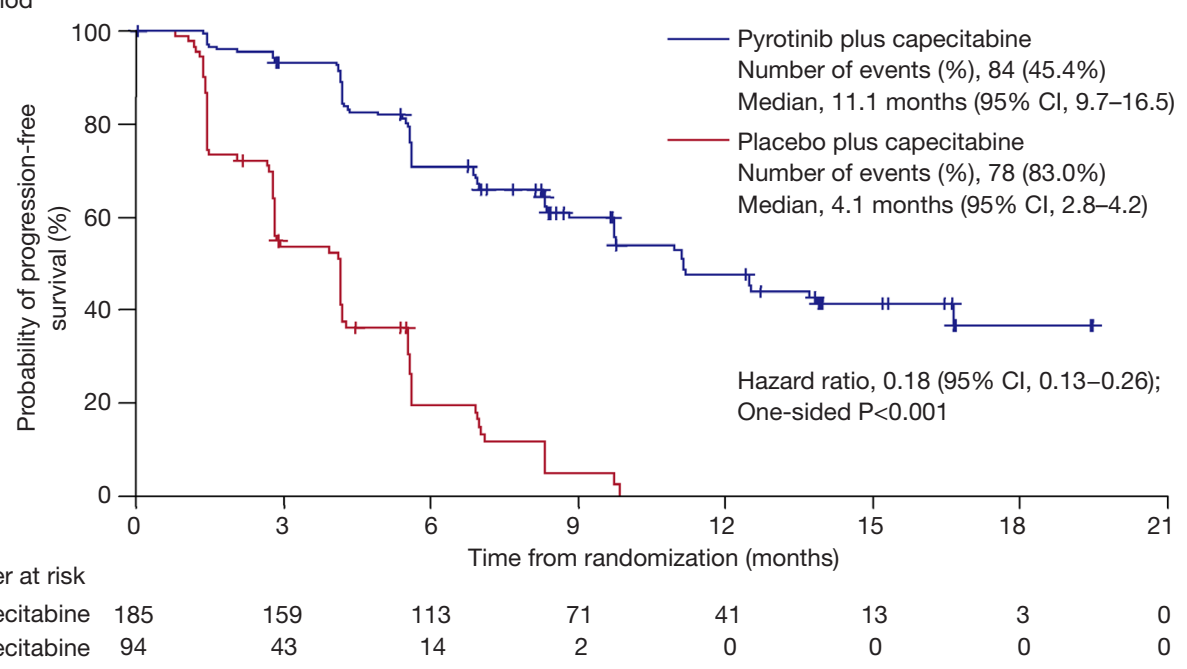

$\begin{array}{ccccccccc}\text { Pyrotinib plus capecitabine } & 185 & 159 & 113 & 71 & 41 & 13 & 3 & 0 \\ \text { Placebo plus capecitabine } & 94 & 43 & 14 & 2 & 0 & 0 & 0 & 0\end{array}$

B

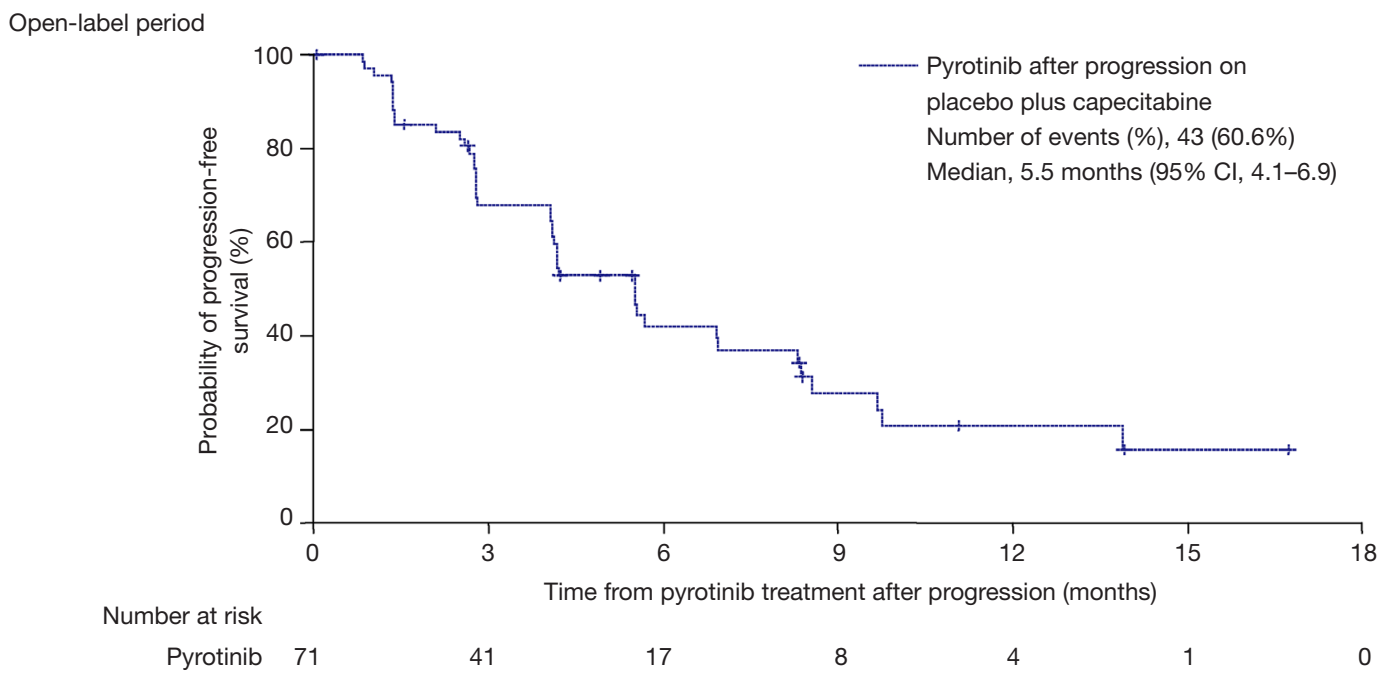

Figure 2 Kaplan-Meier estimates of progression-free survival per independent review committee during the double-blind period (A) and open-label pyrotinib monotherapy period (B). CI, confidence interval.

4.2 months (95\% CI, 0.8-6.9); HR, 0.32 (95\% CI, 0.130.77); $\mathrm{P}=0.011]$ and those without [11.1 months (95\% CI, 9.7-16.5) vs. 4.1 months (95\% CI, 2.8-4.1); HR, 0.17 (95\% CI, 0.12-0.25); $\mathrm{P}<0.001$ ] (Figure S2). In the subpopulation without baseline brain metastases, $1.2 \%$ and $3.6 \%$ of patients in the pyrotinib and placebo groups, respectively, developed new brain metastases, and median time to new brain metastases was 397.5 and 132.0 days, respectively (Table S4). For the subpopulation with untreated brain metastases at baseline, $73.3 \%$ and $87.5 \%$ of patients in the pyrotinib and placebo group, respectively, had progressive brain metastases, and time to progression of brain metastases was 168.0 and 127.0 days, respectively (Table S4).

\section{Safety}

The median number of study-treatment cycles per patient was higher for patients treated with pyrotinib plus capecitabine compared with those with placebo plus capecitabine $[12$ (range, 1-30) vs. 6 (range, 1-20) cycles; Table S5].

Treatment-related adverse events (TRAEs) of any grade occurred in 184 of the 185 patients $(99.5 \%)$ in the pyrotinib group, similar with 90 of the 94 patients $(95.7 \%)$ in the placebo group. The most common TRAEs with an incidence higher than $25.0 \%$ were diarrhea [182 patients $(98.4 \%)$ in the pyrotinib group vs. 64 patients $(68.1 \%)$ in 
Table 2 Tumour response per IRC during double-blind period and open-label pyrotinib monotherapy period

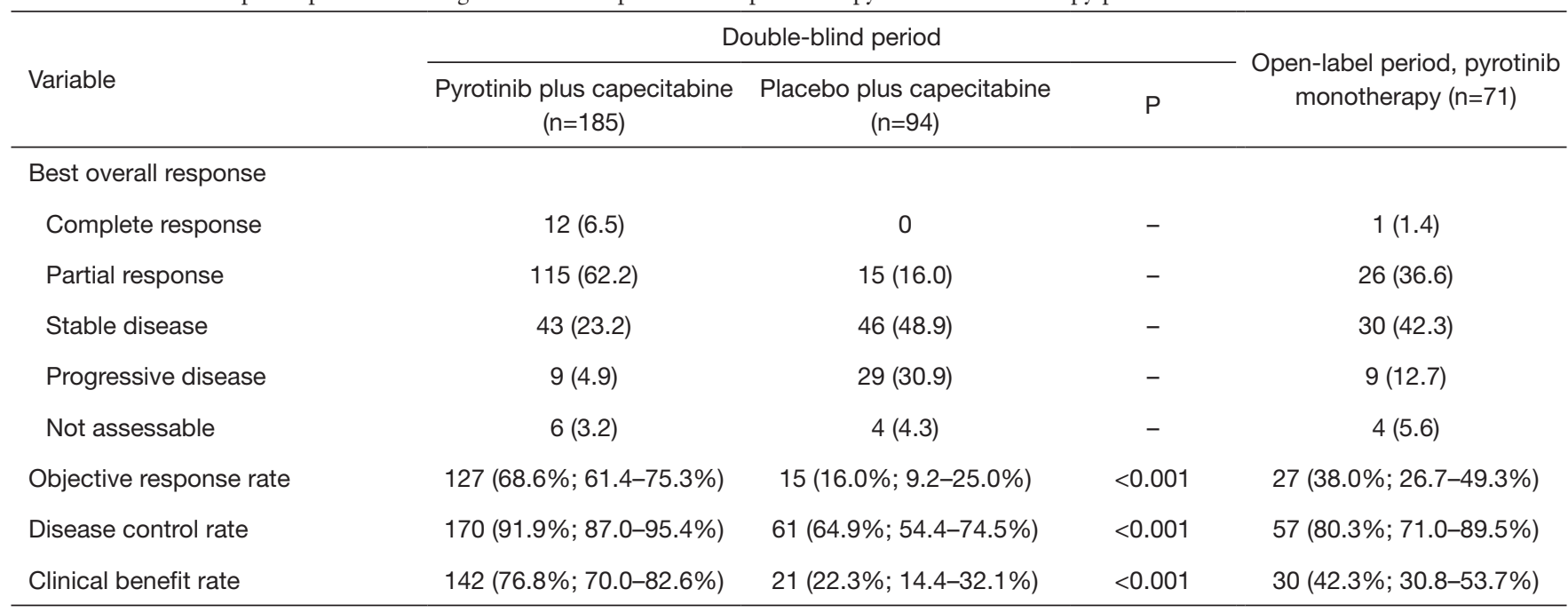

Data are $\mathrm{n}(\%), \mathrm{n}(\% ; 95 \% \mathrm{Cl})$, or median $(95 \% \mathrm{Cl})$. Cl, confidence interval; IRC, independent review committee.

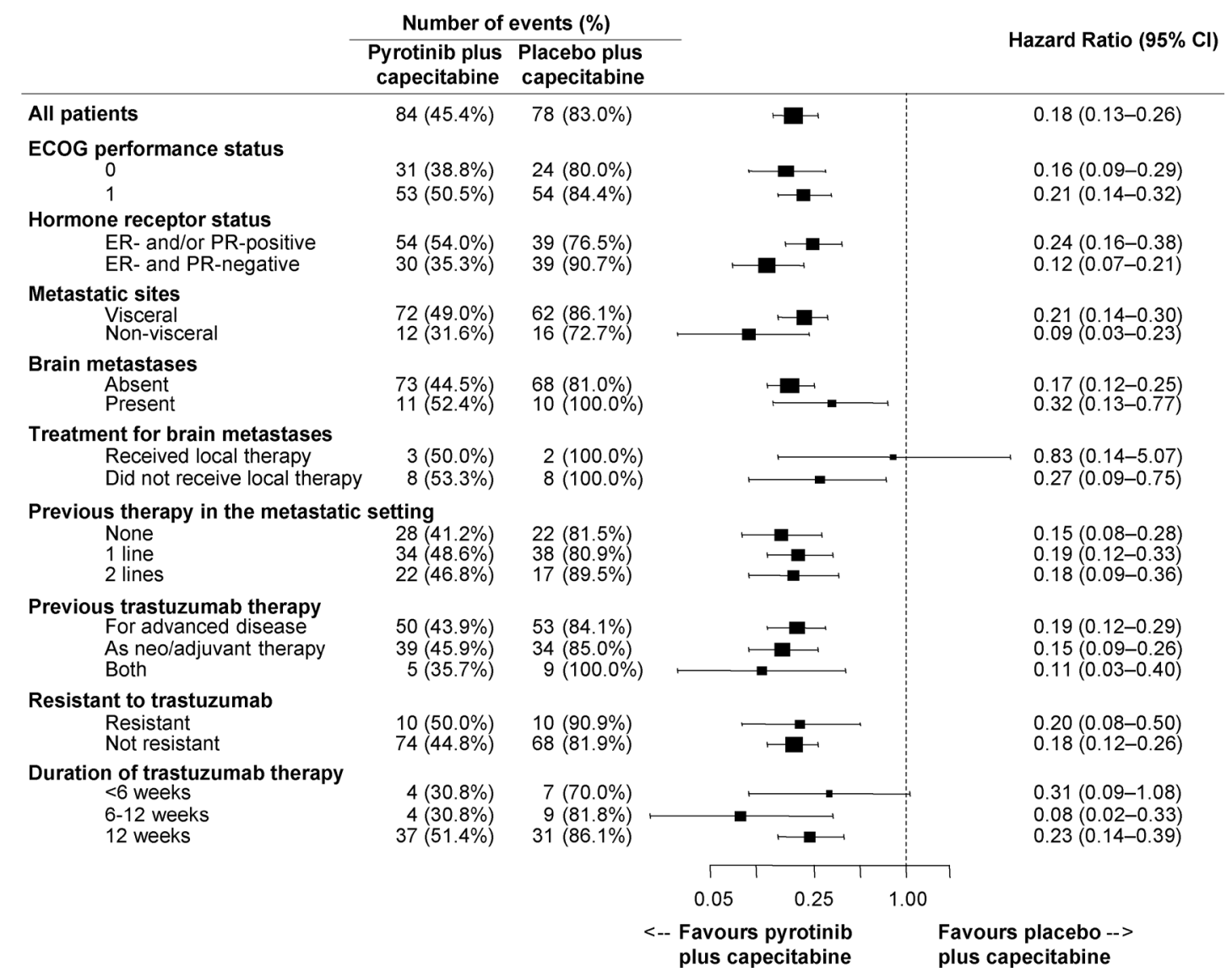

Figure 3 Subgroup analyses of progression-free survival. The dashed line indicates a hazard ratio of 1.00 - the null hypothesis value. Resistance to trastuzumab was defined as having relapsed within 6 months after adjuvant trastuzumab and/or progressed within three months of trastuzumab treatment for metastatic disease. ECOG, Eastern Cooperative Oncology Group; ER, estrogen receptor; PR, progesterone receptor; CI, confidence interval. 
Table 3 Treatment-related adverse events occurring in at least $10 \%$ of patients in either study group during the double-blind period or openlabel pyrotinib monotherapy period

\begin{tabular}{|c|c|c|c|c|c|c|}
\hline \multirow[b]{2}{*}{ Adverse events } & \multicolumn{4}{|c|}{ Double-blind period } & \multirow{2}{*}{\multicolumn{2}{|c|}{$\begin{array}{l}\text { Open-label period, pyrotinib } \\
\text { monotherapy }(n=71)\end{array}$}} \\
\hline & \multicolumn{2}{|c|}{$\begin{array}{l}\text { Pyrotinib plus capecitabine } \\
\qquad(\mathrm{n}=185)\end{array}$} & \multicolumn{2}{|c|}{$\begin{array}{l}\text { Placebo plus capecitabine } \\
\qquad(\mathrm{n}=94)\end{array}$} & & \\
\hline Diarrhea & $182(98.4)$ & $57(30.8)$ & $64(68.1)$ & $12(12.8)$ & $63(88.7)$ & $16(22.5)$ \\
\hline Vomiting & $90(48.6)$ & $4(2.2)$ & $15(16.0)$ & $1(1.1)$ & $12(16.9)$ & $1(1.4)$ \\
\hline White blood cell decreased & $84(45.4)$ & $7(3.8)$ & $28(29.8)$ & $2(2.1)$ & $17(23.9)$ & $1(1.4)$ \\
\hline Aspartate aminotransferase increased & $71(38.4)$ & $2(1.1)$ & $27(28.7)$ & $1(1.1)$ & $16(22.5)$ & $1(1.4)$ \\
\hline Anemia & $56(30.3)$ & $4(2.2)$ & $5(5.3)$ & 0 & $7(9.9)$ & 0 \\
\hline Blood bilirubin increased & $53(28.6)$ & $2(1.1)$ & $25(26.6)$ & $2(2.1)$ & $4(5.6)$ & 0 \\
\hline Weight loss & $48(25.9)$ & $1(0.5)$ & $5(5.3)$ & 0 & $7(9.9)$ & 0 \\
\hline Appetite loss & $46(24.9)$ & $1(0.5)$ & $13(13.8)$ & 0 & $6(8.5)$ & $1(1.4)$ \\
\hline Hypokalemia & $43(23.2)$ & $5(2.7)$ & $3(3.2)$ & $1(1.1)$ & 0 & 0 \\
\hline Pigmentation disorder & $40(21.6)$ & 0 & $13(13.8)$ & 0 & $9(12.7)$ & 0 \\
\hline Bilirubin conjugated increased & 35 (18.9) & 0 & $18(19.1)$ & $1(1.1)$ & 0 & 0 \\
\hline
\end{tabular}

Data are $\mathrm{n}(\%)$. There were no grade 5 treatment-related adverse events.

the placebo group], hand-foot syndrome [110 (59.5\%) vs. $28(29.8 \%)]$, nausea [90 (48.6\%) vs. $17(18.1 \%)]$, vomiting [90 (48.6\%) vs. $15(16.0 \%)]$, decreased white blood cell [84 (45.4\%) vs. 28 (29.8\%)], increased aspartate aminotransferase [71 (38.4\%) vs. 27 (28.7\%)], decreased neutrophil count [68 (36.8\%) vs. 25 (26.6\%)], increased alanine aminotransferase [66 (35.7\%) vs. 21 (22.3\%)], oral mucositis [56 (30.3\%) vs. $12(12.8 \%)$, anemia [56 (30.3\%) vs. $5(5.3 \%)$ ], increased blood bilirubin [53 (28.6\%) vs. 25 (26.6\%)], and weight loss [48 (25.9\%) vs. 5 (5.3\%)] (Table 3). No unexpected TRAEs were found.
Grade 3 or 4 TRAEs occurred in 102 of the 185 patients $(55.1 \%)$ in the pyrotinib group, compared with 24 of the 94 patients $(25.5 \%)$ in the placebo group. The most common ones in the pyrotinib or placebo groups were diarrhea [57 (30.8\%) vs. 12 (12.8\%); all grade 3] and hand-foot syndrome [29 (15.7\%) vs. 5 (5.3\%); all grade 3; Table 3]. The incidence of serious TRAEs was similar in the pyrotinib and placebo group [ 9 of 185 (4.9\%) patients and 4 of 94 (4.3\%), respectively]. Those which occurred in more than $2 \%$ of the patients in either group were diarrhea [4 $(2.2 \%)$ in the pyrotinib group $v s$. none in the placebo 
group], lung infection [none vs. $2(2.1 \%)$ ], and increased blood bilirubin [none vs. 2 (2.1\%)] (Table S6). Four deaths $(2.2 \%)$ in the pyrotinib group occurred within 28 days after study treatment, three due to progression of breast cancer and one due to respiratory failure, but none of them had causal relationship with the study treatments. No patients in the placebo group died during or within 28 days after study treatment.

In the open-label pyrotinib monotherapy period, median number of treatment cycles was 6 (range, 0-24) (Table S5). Grade 3 or 4 TRAEs occurred in 22 patients $(31.0 \%)$. The only grade 3 or 4 TRAE occurring in more than $10 \%$ of patients was diarrhea [16 patients (22.5\%); Table 2]. No serious TRAEs were reported. One death (1.4\%) occurred within 28 days after pyrotinib monotherapy due to respiratory failure and circulatory collapse, which was deemed unrelated to study treatment.

The highest grade of diarrhea irrespective of attribution to treatment was grade 3 . In the pyrotinib group, $24.3 \%$ of patients had grade 3 diarrhea during the first cycle of treatment; the incidence gradually declined during the next 6 cycles, and generally maintained a low level until cycle 20 (Figure S3). In the placebo group, $1.1 \%$ of patients had grade 3 diarrhea during the first cycle of treatment; there was no association between the incidence and treatment cycle. The median time to onset of grade 3 diarrhea was 8.0 days in the pyrotinib group $v s .135 .5$ days in the placebo group. The incidence and median cumulative duration of grade 3 diarrhea were $31.4 \%(58 / 185)$ and 9.0 days in the pyrotinib group and $12.8 \%$ (12/94) and 15.0 days in the placebo group. Only one patient discontinued pyrotinib due to diarrhea.

\section{Discussion}

Compared with placebo plus capecitabine, pyrotinib plus capecitabine significantly improved the PFS (11.1 vs. 4.1 months; $\mathrm{P}<0.001$ ) and reduced the risk of progression or death by $82 \%$ in patients after prior trastuzumab and taxanes. Therefore, this study met its primary endpoint at an adjusted significant level of $\leq 0.0043$.

Multiple studies have investigated treatment strategies following trastuzumab, but not other anti-HER2 agents (Table S7). Lapatinib plus capecitabine had a PFS of 6.4-6.8 months and an ORR of $22-41 \%(18-20)$. The PFS with neratinib plus capecitabine was 35.9 weeks (approximately 8.3 months) for patients previously treated with lapatinib and 40.3 weeks (approximately 9.3 months) for those with no prior lapatinib, and the ORR was 57\% and $64 \%$, respectively (21). Lapatinib plus trastuzumab only had a PFS of 12.0 weeks, and ORR was $10.3 \%$ (22). In the EMILIA study, T-DM1 monotherapy achieved a median PFS of 9.6 months and an ORR of $43.6 \%$ (19). We found that a combination of pyrotinib with capecitabine showed clinically significant PFS benefit in HER2-positive metastatic breast cancer patients who had previously received trastuzumab and taxanes. In addition, a phase 2 study of pyrotinib plus capecitabine showed significantly higher ORR and prolonged PFS compared with lapatinib plus capecitabine in pre-treated HER2-positive metastatic breast cancer (23). In the Chinese Society of Clinical Oncology (CSCO) 2020 guideline for breast cancer, pyrotinib combined with capecitabine was added as a level I recommendation for HER2-positive metastatic breast cancer patients resistant to trastuzumab (24).

Studies evaluating monotherapies with small-molecule anti-HER2 TKIs showed that the median PFS was 8.1 weeks (about 1.9 months) with lapatinib and 4.5 month/22.3 weeks (about 5.1 months) with neratinib, and the ORR was $6.9 \%$ and $24 \% / 29 \%$, respectively $(18,22,25)$. In this study, we designed sequential pyrotinib monotherapy for patients in the control group. A median PFS of 5.5 months and an ORR of $38.0 \%$ were achieved, suggesting the potent efficacy of pyrotinib alone. However, given that the median PFS with pyrotinib plus capecitabine was up to 11.1 months and the ORR was as high as $68.6 \%$, we still recommend the combination of pyrotinib plus capecitabine as the treatment option after trastuzumab. Also, the high objective response and long survival benefit of pyrotinib alone provides a basis for maintenance therapy or combination therapy of pyrotinib with other drugs. Based on our findings, the CSCO 2020 guideline for breast cancer has added pyrotinib monotherapy as a level III recommendation for HER2-positive metastatic breast cancer patients resistant to trastuzumab (24).

With prolonged survival, HER2-positive breast cancer patients are at high risk for central nervous system (CNS) metastases. More than 35\% patients with HER2-positive breast cancer developed metastatic brain disease $(26,27)$. However, treatment options are limited, involving mainly local brain surgery or radiotherapy. The phase 2 LANDSCAPE study showed that lapatinib plus capecitabine in patients with previously untreated brain metastases achieved a high ORR of $65.9 \%$ (28). In the phase 3 NALA study, some HER2-positive metastatic breast cancer patients with asymptomatic and stable brain 
metastases were enrolled. Incidence of intervention for CNS metastases was significantly reduced from $29.2 \%$ in the lapatinib plus capecitabine group to $22.8 \%$ in the neratinib plus capecitabine group $(\mathrm{P}=0.043)$ (29). In this study, there were 31 patients with brain metastases. They also could benefit from treatment with pyrotinib combined with capecitabine (median PFS 6.9 vs. 4.2 months, HR 0.32, $\mathrm{P}<0.001$ ), but future studies are warranted to confirm these results.

The most common AE related to pyrotinib plus capecitabine treatment was diarrhea. Primary prophylaxis for diarrhea was not prespecified. Grade 3 treatmentrelated diarrhea occurred in $30.8 \%$ of patients with pyrotinib plus capecitabine and $22.5 \%$ with pyrotinib monotherapy $(18,25)$. It occurred mostly during the first treatment cycle, with $50 \%$ of patients experiencing diarrhea between days 1-10. Despite the high incidence, diarrhea was generally reversible with anti-diarrhea treatment, treatment interruption, or dose reduction, and barely led to discontinuation of either study treatment. Early treatment after diarrhea could effectively reduce the incidence of grade 3 diarrhea. As the study progressed, the incidence of diarrhea showed a decreasing trend in patients with pyrotinib plus capecitabine, but not in those with placebo plus capecitabine. Doctor and patient education are important in the management of AEs. Patients are instructed to interrupt capecitabine if there is persistent grade 3 diarrhea, or grade 1-2 diarrhea with complications (grade 2 nausea, vomiting, fever, hematochezia, or dehydration) and to start anti-diarrhea treatment with loperamide or montmorillonite powder as early as possible. If diarrhea does not resolve 3 days after withholding capecitabine, patients should interrupt pyrotinib treatment until diarrhea resolves to grade $0-1$.

A limitation of this study was that the control group was not the standard second-line therapy in China, as we tried to mimic a real-world scenario. Secondly, the HER2 status of patients was not centralized confirmed. Besides, OS data for the study remain unavailable, requiring further follow-up.

\section{Conclusions}

This study proves the substantial clinical benefit and manageable safety of pyrotinib plus capecitabine in patients with HER2-positive relapsed or metastatic breast cancer after trastuzumab, as compared with placebo plus capecitabine. Pyrotinib plus capecitabine offers a potent treatment option for these patients, especially for resourcelimited regions or populations. Patients who progressed on capecitabine therapy could still benefit from sequential pyrotinib monotherapy. Pyrotinib and capecitabine also shows potential efficacy in patients with CNS metastases.

\section{Acknowledgments}

We thank all patients and their families, investigators (Yuee Teng, Jing Cheng, Yueyin Pan, Peifen Fu, Yanxia Shi, Ting Luo, Yunjiang Liu, Yongsheng Wang, Hongyuan Li, Kun Wang, and Shu Wang; Table S1), and research personnel for participating in this study. Data analysis was conducted by statisticians from Tigermed Consulting Co., Ltd. (Hangzhou China). Writing assistance according to Good Publication Practice Guidelines was provided by Hui Dong, PhD (Senior Medical Writer at Hengrui). We thank Tao Wang, PhD (Senior Director, Statistics and Programming at Hengrui) for his input in statistical review. We would also like to acknowledge Editage for language editing.

Funding: This study was sponsored by Jiangsu Hengrui Medicine Co., Ltd.

\section{Footnote}

Reporting Checklist: The authors have completed the CONSORT reporting checklist. Available at http://dx.doi. org/10.21037/tbcr-20-25

Data Sharing Statement: Available at http://dx.doi. org/10.21037/tbcr-20-25

Conflicts of Interest: All authors have completed the ICMJE uniform disclosure form (available at http://dx.doi. org/10.21037/tbcr-20-25). ZJ serves as an unpaid Editor-inChief of Translational Breast Cancer Research. QZ, YY, XW serve as unpaid editorial board member of Translational Breast Cancer Research from Mar 2020 to Feb 2022. JZ and XZ are employed by Jiangsu Hengrui Medicine Co., Ltd. The other authors have no conflicts of interest to declare.

Ethical Statement: The authors are accountable for all aspects of the work in ensuring that questions related to the accuracy or integrity of any part of the work are appropriately investigated and resolved. The protocol and all amendments were approved by the Ethics Committee of each participating site (Table S2). The study was conducted in accordance with Helsinki Declaration of 1964 (revised 
2013), Good Clinical Practice, and Chinese laws and regulatory requirements. All patients provided written informed consent.

Open Access Statement: This is an Open Access article distributed in accordance with the Creative Commons Attribution-NonCommercial-NoDerivs 4.0 International License (CC BY-NC-ND 4.0), which permits the noncommercial replication and distribution of the article with the strict proviso that no changes or edits are made and the original work is properly cited (including links to both the formal publication through the relevant DOI and the license). See: https://creativecommons.org/licenses/by-nc-nd/4.0/.

\section{References}

1. Slamon DJ, Godolphin W, Jones LA, et al. Studies of the HER-2/neu proto-oncogene in human breast and ovarian cancer. Science 1989;244:707-12.

2. Eroglu Z, Tagawa T, Somlo G. Human epidermal growth factor receptor family-targeted therapies in the treatment of HER2-overexpressing breast cancer. Oncologist 2014;19:135-50.

3. Slamon DJ, Clark GM, Wong SG, et al. Human breast cancer: correlation of relapse and survival with amplification of the HER-2/neu oncogene. Science 1987;235:177-82.

4. Ross JS, Slodkowska EA, Symmans WF, et al. The HER2 receptor and breast cancer: ten years of targeted antiHER-2 therapy and personalized medicine. Oncologist 2009;14:320-68.

5. Dawood S, Broglio K, Buzdar AU, et al. Prognosis of women with metastatic breast cancer by HER2 status and trastuzumab treatment: an institutional-based review. J Clin Oncol 2010;28:92-8.

6. Gao S, Barber B, Schabert V, et al. Tumor hormone/ HER2 receptor status and pharmacologic treatment of metastatic breast cancer in Western Europe. Curr Med Res Opin 2012;28:1111-8.

7. Yardley DA, Kaufman PA, Brufsky A, et al. Treatment patterns and clinical outcomes for patients with de novo versus recurrent HER2-positive metastatic breast cancer. Breast Cancer Res Treat 2014;145:725-34.

8. Blackwell K, Gligorov J, Jacobs I, et al. The Global Need for a Trastuzumab Biosimilar for Patients With HER2-Positive Breast Cancer. Clin Breast Cancer 2018;18:95-113.

9. Li J, Wang S, Wang Y, et al. Disparities of Trastuzumab
Use in Resource-Limited or Resource-Abundant Regions and Its Survival Benefit on HER2 Positive Breast Cancer: A Real-World Study from China. Oncologist 2017;22:1333-8.

10. Sung M, Tan X, Lu B, et al. Caveolae-Mediated Endocytosis as a Novel Mechanism of Resistance to Trastuzumab Emtansine (T-DM1). Mol Cancer Ther 2018;17:243-53.

11. D'Amato V, Raimondo L, Formisano L, et al. Mechanisms of lapatinib resistance in HER2-driven breast cancer. Cancer Treat Rev 2015;41:877-83.

12. Vernieri C, Milano M, Brambilla M, et al. Resistance mechanisms to anti-HER2 therapies in HER2-positive breast cancer: Current knowledge, new research directions and therapeutic perspectives. Crit Rev Oncol Hematol 2019;139:53-66.

13. Li X, Yang C, Wan H, et al. Discovery and development of pyrotinib: A novel irreversible EGFR/HER2 dual tyrosine kinase inhibitor with favorable safety profiles for the treatment of breast cancer. Eur J Pharm Sci 2017;110:51-61.

14. Gourd E. Pyrotinib shows activity in metastatic breast cancer. Lancet Oncol 2017;18:e643.

15. Ma F, Li Q, Chen S, et al. Phase I Study and Biomarker Analysis of Pyrotinib, a Novel Irreversible Pan-ErbB Receptor Tyrosine Kinase Inhibitor, in Patients With Human Epidermal Growth Factor Receptor 2-Positive Metastatic Breast Cancer. J Clin Oncol 2017;35:3105-12.

16. Li Q, Guan X, Chen S, et al. Safety, Efficacy and Biomarker Analysis of Pyrotinib in Combination with Capecitabine in HER2-Positive Metastatic Breast Cancer Patients: A Phase I Clinical Trial. Clin Cancer Res 2019;25:5212-20.

17. Wolff AC, Hammond ME, Hicks DG, et al. Recommendations for human epidermal growth factor receptor 2 testing in breast cancer: American Society of Clinical Oncology/College of American Pathologists clinical practice guideline update. J Clin Oncol 2013;31:3997-4013.

18. Martin M, Bonneterre J, Geyer CE Jr, et al. A phase two randomised trial of neratinib monotherapy versus lapatinib plus capecitabine combination therapy in patients with HER2 + advanced breast cancer. Eur J Cancer 2013;49:3763-72.

19. Verma S, Miles D, Gianni L, et al. Trastuzumab emtansine for HER2-positive advanced breast cancer. N Engl J Med 2012;367:1783-91.

20. Kaufman B, Trudeau M, Awada A, et al. Lapatinib 
monotherapy in patients with HER2-overexpressing relapsed or refractory inflammatory breast cancer: final results and survival of the expanded HER2 + cohort in EGF103009, a phase II study. Lancet Oncol 2009;10:581-8.

21. Saura C, Garcia-Saenz JA, Xu B, et al. Safety and efficacy of neratinib in combination with capecitabine in patients with metastatic human epidermal growth factor receptor 2-positive breast cancer. J Clin Oncol 2014;32:3626-33.

22. Blackwell KL, Burstein HJ, Storniolo AM, et al. Randomized study of Lapatinib alone or in combination with trastuzumab in women with ErbB2-positive, trastuzumab-refractory metastatic breast cancer. J Clin Oncol 2010;28:1124-30.

23. Ma F, Ouyang Q, Li W, et al. Pyrotinib or Lapatinib Combined With Capecitabine in HER2-Positive Metastatic Breast Cancer With Prior Taxanes, Anthracyclines, and/or Trastuzumab: A Randomized, Phase II Study. J Clin Oncol 2019;37:2610-9.

24. Li J, Jiang Z. CSCO BC guideline: updates for HER2 positive breast cancer in 2020. Transl Breast Cancer Res 2020;1:4.

25. Burstein HJ, Sun Y, Dirix LY, et al. Neratinib, an irreversible ErbB receptor tyrosine kinase inhibitor, in

doi: $10.21037 /$ tbcr-20-25

Cite this article as: Yan M, Bian L, Hu X, Zhang Q, Ouyang Q, Feng J, Yin Y, Sun T, Tong Z, Wang X, Yao H, Zou J, Zhu $X$, Jiang Z. Pyrotinib plus capecitabine for human epidermal growth factor receptor 2-positive metastatic breast cancer after trastuzumab and taxanes (PHENIX): a randomized, doubleblind, placebo-controlled phase 3 study. Transl Breast Cancer Res 2020;1:13. patients with advanced ErbB2-positive breast cancer. J Clin Oncol 2010;28:1301-7.

26. Brufsky AM, Mayer M, Rugo HS, et al. Central nervous system metastases in patients with HER2-positive metastatic breast cancer: incidence, treatment, and survival in patients from registHER. Clin Cancer Res 2011;17:4834-43.

27. Gori S, Rimondini S, De Angelis V, et al. Central nervous system metastases in HER-2 positive metastatic breast cancer patients treated with trastuzumab: incidence, survival, and risk factors. Oncologist 2007;12:766-73.

28. Bachelot T, Romieu G, Campone M, et al. Lapatinib plus capecitabine in patients with previously untreated brain metastases from HER2-positive metastatic breast cancer (LANDSCAPE): a single-group phase 2 study. Lancet Oncol 2013;14:64-71.

29. Saura C, Oliveira M, Feng YH, et al. Neratinib + capecitabine versus lapatinib + capecitabine in patients with HER2 + metastatic breast cancer previously treated with $\geq 2$ HER2-directed regimens: Findings from the multinational, randomized, phase III NALA trial. J Clin Oncol 2019;37:abstr 1002. 


\section{Full inclusion and exclusion criteria}

\section{Inclusion criteria}

Subjects must fulfill all of the following inclusion criteria to be eligible for this trial:

(I) Aged 18 to 75 years, female patients;

(II) ECOG performance status of 0-1;

(III) Life expect more than 12 weeks;

(IV) With at least one measurable lesion according to RECIST 1.1 criteria, and has progressed after or during the last antitumor treatment;

(V) Pathologically confirmed HER2-positive recurrent/metastatic breast cancer;

(i) Positive HER2 is defined as those with grade 3+ staining intensity by immunohistochemical analysis and/or HER2 gene amplification by fluorescence in-situ hybridization (FISH) (reviewed and confirmed by the investigators at the study site).

(VI) Patients who have progressed disease during or after treatment with trastuzumab, and are not amenable or available for trastuzumab or lapatinib treatment;

(i) Consecutive use of trastuzumab for $\geq 2$ cycles in recurrence/metastasis setting, or

(ii) Recurrence/metastasis after consecutive use of trastuzumab for $\geq 3$ months in adjuvant setting.

(VII) Prior treatment with taxanes;

(VIII) Prior $\leq 2$ lines of chemotherapy in recurrence/metastasis setting;

(IX) The function of main organs must meet the following requirements (no blood transfusion within 2 weeks prior to screening, no use of drugs to increase white blood cell or platelet):

(i) Routine blood test:

* Absolute neutrophil count (ANC) $\geq 1.5 \times 10^{9} / \mathrm{L}$;

* Platelet count (PLT) $\geq 90 \times 10^{9} / \mathrm{L}$;

* Haemoglobin $(\mathrm{Hb}) \geq 90 \mathrm{~g} / \mathrm{L}$.

(ii) Chemistry:

* Total bilirubin (TBIL) $\leq 1.5 \times$ upper limit of normal (ULN);

* Alanine aminotransferase (ALT) or aspartate aminotransferase (AST) $\leq 2 \times \mathrm{ULN}$, or $\leq 5 \times \mathrm{ULN}$ in the presence of liver metastases;

* Urea nitrogen (BUN) and creatinine $(\mathrm{Cr}) \leq 1.5 \times \mathrm{ULN}$.

(iii) Echocardiography:

* Left ventricular ejection fraction (LVEF) $\geq 50 \%$.

(iv) 12-lead ECG:

* Fridericia-corrected QT interval (QTcF) $<470 \mathrm{~ms}$.

(X) Being voluntary to participate in the study, sign the informed consent form, with good compliance and willingness to cooperate with follow-up.

\section{Exclusion criteria}

Subject will not be included if any of the following conditions is met:

(I) Patients with brain metastases that are symptomatic and require treatment;

(II) Previous use of capecitabine (those who have used capecitabine during adjuvant therapy and discontinued it for $\geq 6$ months are allowed to be enrolled);

(III) Use of chemotherapy, targeted therapy or investigational product within 4 weeks prior to randomization; use of endocrine therapy within 7 days prior to randomization;

(IV) Previous use of tyrosine kinase inhibitor targeting HER2 (including lapatinib, pyrotinib and neratinib);

(V) The 3rd space effusion (e.g., hydrothorax and ascites) that cannot be controlled by drainage or other method;

(VI) Inability to swallow, bowel obstruction, or presence of other factors affecting drug intake and absorption; 
(VII) Known history of allergy to the drug components in this protocol; history of immunodeficiency disease, including positive HIV test, or other acquired, congenital immunodeficiency disease, or history of organ transplantation;

(VIII) Other malignant tumors in the past 5 years, exception of cured carcinoma cervix in situ, basal cell or squamous cell carcinoma of skin;

(IX) Pregnant or lactating female patient, female patient with childbearing potential and positive pregnancy test at baseline, or the females of childbearing potential who are not willing to use effective contraceptive measures throughout the trial;

(X) Severe concurrent disease, or any other condition that is considered by investigators as unsuitable to participate in this study.

\section{Protocol pre-specified algorithm for management of diarrhea as follows:}

Before taking the study drug, patients should be informed by investigators regarding the possibility of diarrhea and corresponding treatment measures. Symptomatic treatment such as loperamide (initially $4 \mathrm{mg}$ followed by $2 \mathrm{mg}$ after each unformed stool, up to a maximum of $16 \mathrm{mg}$ daily) or Montmorillonite powder ( $3 \mathrm{~g} / \mathrm{packet}$ tid) should be given when diarrhea occurs, followed by close follow-up or observation ( $\leq 14$ days). Oral or intravenous electrolyte can be given for serious diarrhea. For grade 3 diarrhea that could not be resolved after symptomatic treatments or grade 1 or 2 diarrhea with complications, treatment with capecitabine should be suspended; if the adverse event can still not be controlled after temporary discontinuation of capecitabine, treatment with pyrotinib/placebo should be suspended at the discretion of investigator. The drug can be resumed after the adverse event is recovered to grade 1 or disappears. 
Table S1 Study sites, investigators' names and enrollment for patients in the PHENIX study

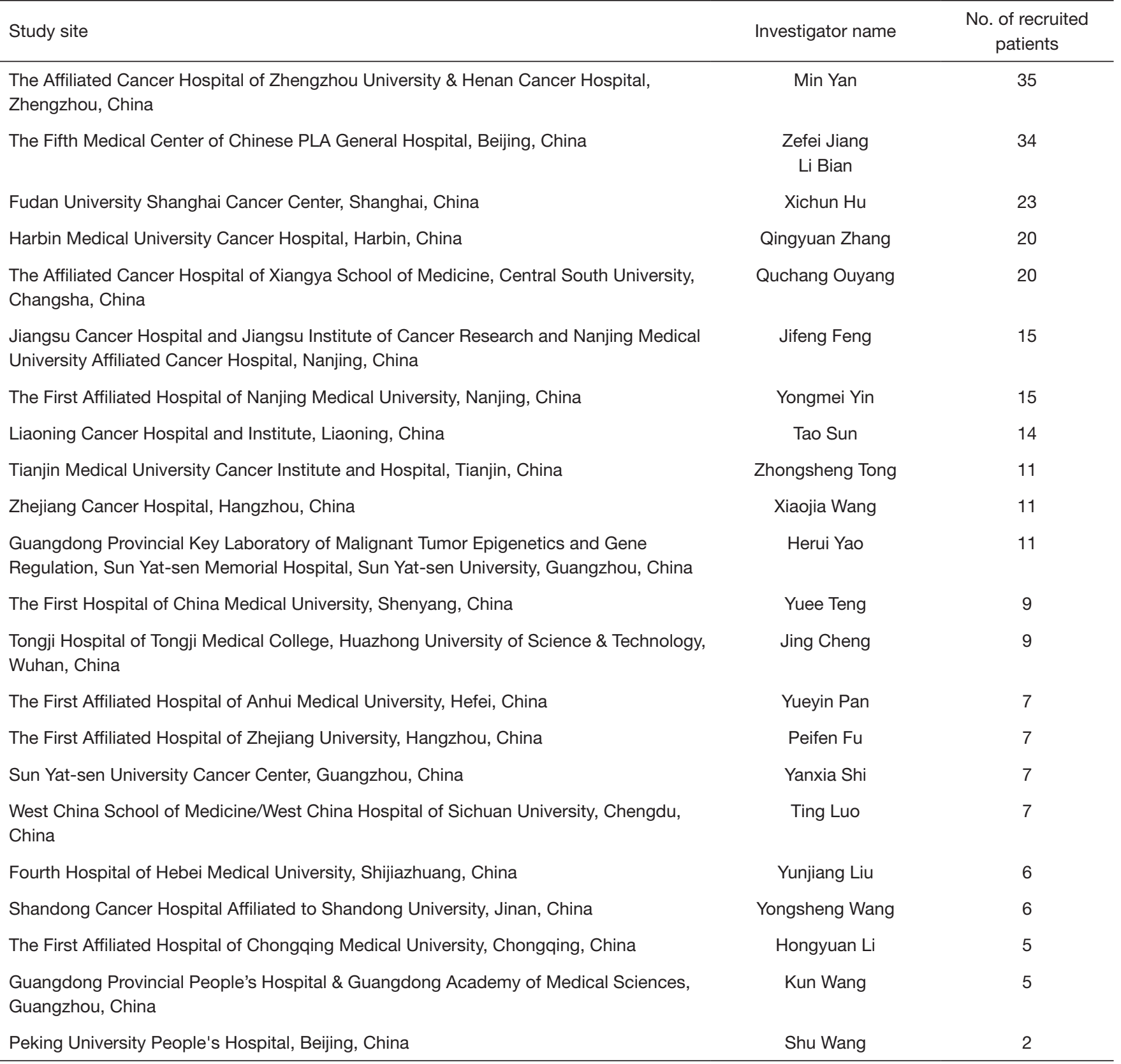


Table S2 Name of the ethics committee of each participating site and the number of the approvals

\begin{tabular}{|c|c|}
\hline Name of ethics committee & Number of the approval \\
\hline Ethics Committee of Peking University People's Hospital & 2016РHA049-01 \\
\hline Ethics Committee of Jiangsu Cancer Hospital & 2016-036 \\
\hline Ethics Committee of The First Affiliated Hospital with Nanjing Medical University & 2016-MD-166 \\
\hline Medical Ethics Committee of Zhejiang Cancer Hospital & IRB-[2016]93 \\
\hline Ethics Committee of Harbin Medical University Cancer Hospital & 2016-39 \\
\hline Medical Ethics Committee of Liaoning Cancer Hospital \& Institute & $20160912-1$ \\
\hline Medical Ethics Committee of The First Hospital of China Medical University & 2016YL025 \\
\hline Clinical Trail Ethics Committee of The Fourth Hospital of Hebei Medical University & 2016017 \\
\hline Medical Ethics Committee of Hunan Cancer Hospital & $2017-25$ \\
\hline Medical Ethics Committee of Henan Cancer Hospital & 2016044 \\
\hline Medical Ethics Committee of Sun Yat-Sen Memorial Hospital, Sun Yat-Sen University & $2016-27$ \\
\hline Medical Ethics Committee of Sun Yat-Sen University Cancer Center & A2017-001-01 \\
\hline Ethics Committee of The First Affiliated Hospital, College of Medicine, Zhejiang University & $2016-100$ \\
\hline Ethics Committee of Clinical Trial of West China Hospital, Sichuan University & $2017-2$ \\
\hline Clinical Trail Ethics Committee of Huazhong University Science and Technology & $2016-139$ \\
\hline
\end{tabular}

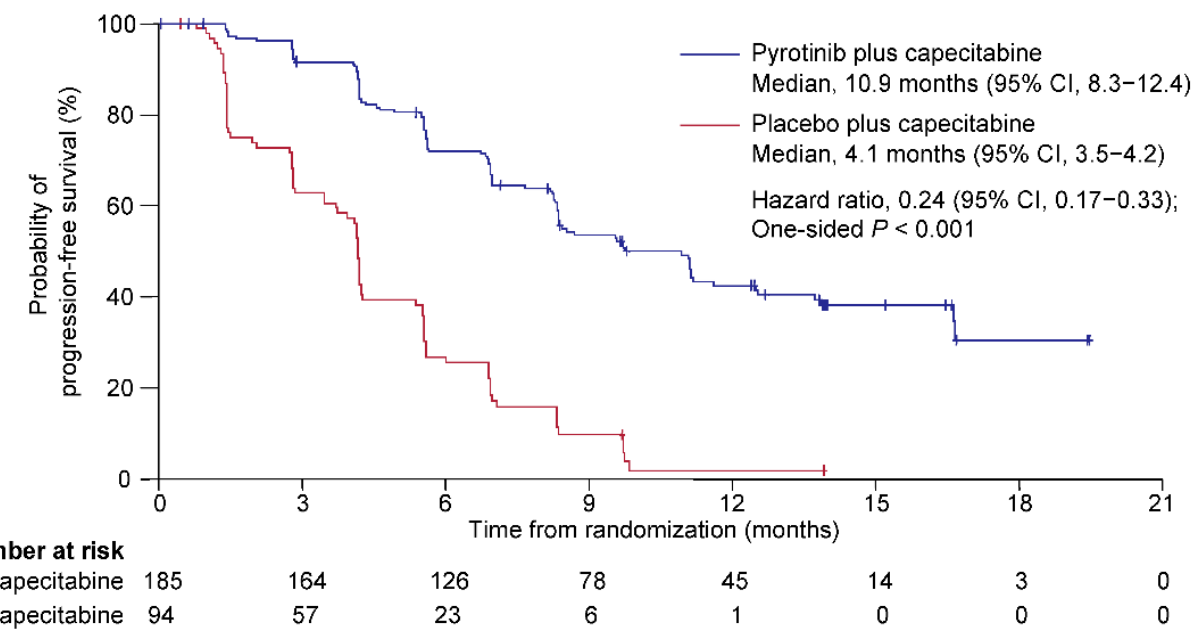

Figure S1 Kaplan-Meier estimates of progression-free survival per investigator during double-blind period. Dashes on the curves represent censored patients. HR, hazard ratio. 
Table S3 Tumour response per investigator during double-blind period

\begin{tabular}{|c|c|c|c|}
\hline Variable & Pyrotinib plus capecitabine $(n=185)$ & Placebo plus capecitabine $(n=94)$ & $\mathrm{P}$ \\
\hline Complete response & $9(4.9)$ & 0 & - \\
\hline Partial response & $124(67.0)$ & $15(16.0)$ & - \\
\hline Stable disease & $43(23.2)$ & $54(57.4)$ & - \\
\hline Not assessable & $3(1.6)$ & $2(2.1)$ & - \\
\hline Objective response rate & 133 (71.9\%; 64.8-78.2\%) & 15 (16.0\%; 9.2-25.0\%) & $<0.001$ \\
\hline Duration of response, months & $11.1(9.49-N R)$ & $5.5(2.79-5.65)$ & $<0.001$ \\
\hline Ongoing responses & $70(52.6)$ & $2(13.3)$ & - \\
\hline
\end{tabular}

Data are $\mathrm{n}(\%), \mathrm{n}(\% ; 95 \% \mathrm{Cl})$, or median $(95 \% \mathrm{Cl})$. Cl, confidence interval; NR, not reached; IRC, independent review committee. 
A Patients with brain metastases at baseline

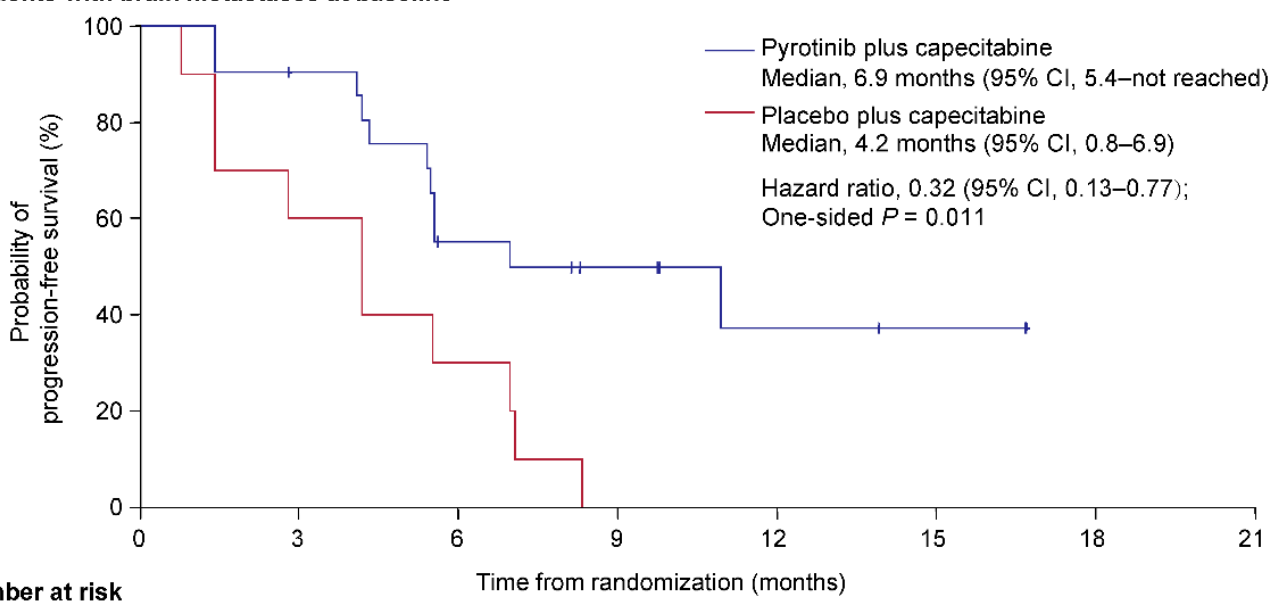

Number at risk

Pyrotinib plus capecitabine

$21 \quad 18$

$18 \quad 10$

6

3

32

20

$0 \quad 0$

Placebo plus capecitabine

10

6

3

0

0

0

0

0

B Patients without brain metastases at baseline

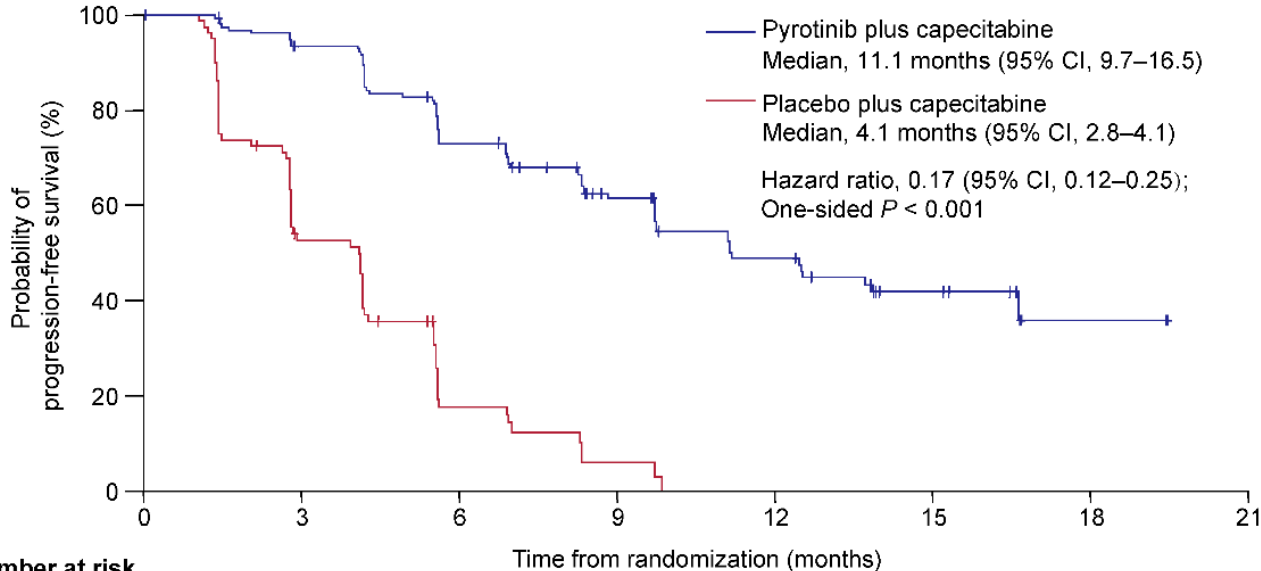

Number at risk

Time from randomization (months)

Pyrotinib plus capecitabine

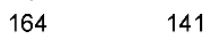

$141 \quad 103$

65

38

11

3

0

Placebo plus capecitabine

$84 \quad 37$

11

2

0

0

0

0

Figure S2 Kaplan-Meier estimates of progression-free survival in patients with (A) and without (B) brain metastases at baseline. CI, confidence interval. 
Table S4 Efficacy in patients with vs. without brain metastases at baseline

\begin{tabular}{|c|c|c|}
\hline Variable & Pyrotinib plus capecitabine $(n=185)$ & Placebo plus capecitabine $(n=94)$ \\
\hline Patients without brain metastases & $1.2(2 / 164)$ & $3.6(3 / 84)$ \\
\hline Patients with brain metastases & $71.4(15 / 21)$ & $90.0(9 / 10)$ \\
\hline Received local therapy & $66.7(4 / 6)$ & $100.0(2 / 2)$ \\
\hline \multicolumn{3}{|c|}{ Median time to progressive brain metastases [range], days } \\
\hline Patients without brain metastases & 397.5 [378-417] & $132.0[127-184]$ \\
\hline Patients with brain metastases & $176.0[85-337]$ & $131.0[27-297]$ \\
\hline Received local therapy & 179.5 [94-212] & $279.0[261-297]$ \\
\hline
\end{tabular}

Proportion was compared using Fisher's exact test, and time to progressive was compared using Log-rank test.

Table S5 Study treatment

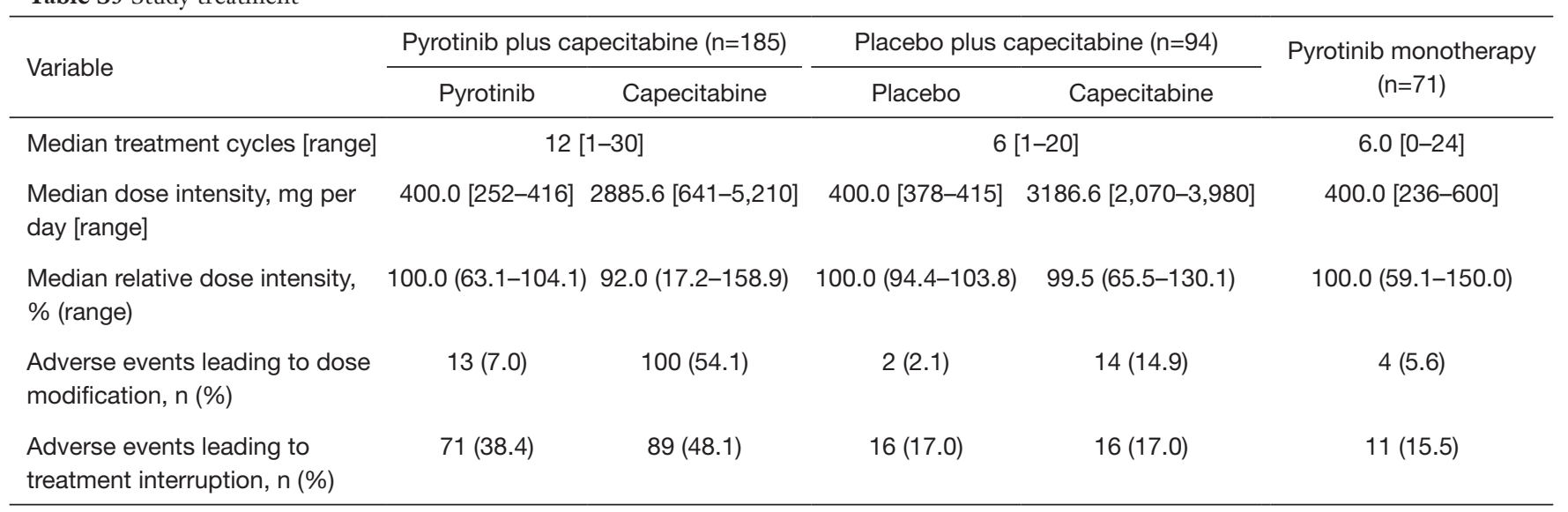


Table S6 All treatment-related serious adverse event

\begin{tabular}{|c|c|c|}
\hline Adverse event & Pyrotinib plus capecitabine $(n=185)$ & Placebo plus capecitabine $(n=94)$ \\
\hline Herpes zoster & $1(0.5 \%)$ & 0 \\
\hline Streptococcal infection & $1(0.5 \%)$ & 0 \\
\hline Gastroenteritis & $1(0.5 \%)$ & 0 \\
\hline Alanine aminotransferase increased & $1(0.5 \%)$ & 0 \\
\hline Aspartate aminotransferase increased & $1(0.5 \%)$ & 0 \\
\hline Vomiting & $1(0.5 \%)$ & 0 \\
\hline Palpitation & $1(0.5 \%)$ & 0 \\
\hline Blood bilirubin increased & 0 & $2(2.1 \%)$ \\
\hline Bilirubin conjugated increased & 0 & $1(1.1 \%)$ \\
\hline Blood bilirubin unconjugated increased & 0 & $1(1.1 \%)$ \\
\hline Bone marrow failure & 0 & $1(1.1 \%)$ \\
\hline
\end{tabular}

No treatment-related serious adverse events were reported during open-label pyrotinib monotherapy period.

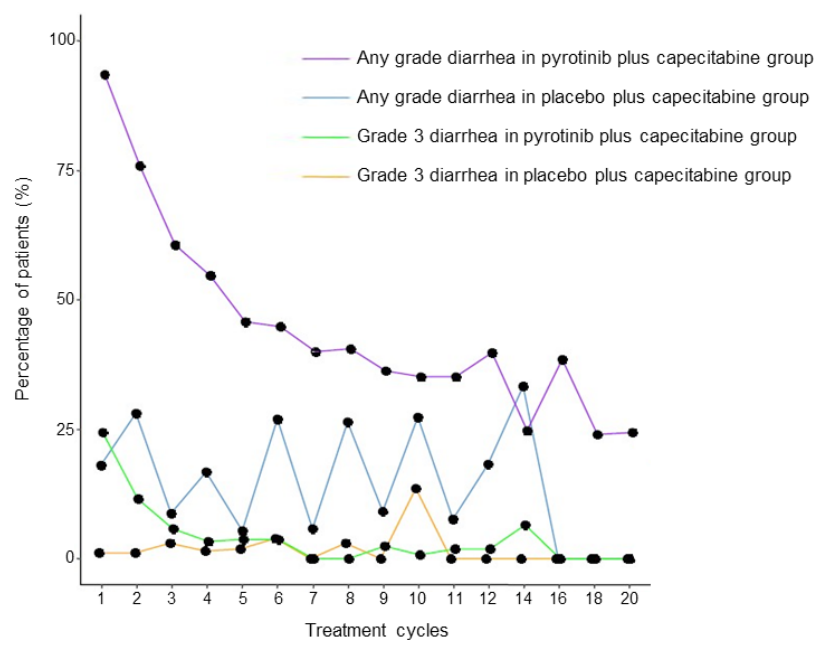

Figure S3 Diarrhea events over time. 
Table S7 Literature search regarding anti-HER2 antibodies in advanced or metastatic breast cancer after trastuzumab-based therapy

\begin{tabular}{|c|c|c|c|c|c|c|c|}
\hline TKI & Year & Study & Population & Design & Intervention and sample size & ORR & PFS \\
\hline $\begin{array}{l}\text { Lapatinib/lapatinib + } \\
\text { trastuzumab }\end{array}$ & 2006 & $\begin{array}{l}\text { EGF104900, } \\
\text { NCT00320385 }\end{array}$ & $\begin{array}{l}\text { HER2-positive metastatic breast cancer patients; progression on prior trastuzumab- } \\
\text { based therapy }\end{array}$ & $\begin{array}{l}\text { Phase III, randomized, multicenter, open- } \\
\text { label study }\end{array}$ & Lapatinib + trastuzumab (n=148), lapatinib (n=148) & $10.3 \%, 6.9 \% ; P=0.46$ & 12.0 weeks, 8.1 weeks; $H R=0.73 ; P=0.008$ \\
\hline \multirow[t]{2}{*}{ Neratinib } & 2006 & NCT00300781 & HER2-positive advanced or metastatic breast cancer patients w/o prior trastuzumab & Randomized, open-label, phase II & Neratinib (prior trastuzumab, $\mathrm{n}=66$; no prior trastuzumab, $\mathrm{n}=70$ ) & $\begin{array}{l}\text { Trastuzumab-treated: } 24 \% \text {; } \\
\text { trastuzumab-naive: } 56 \%\end{array}$ & $\begin{array}{l}\text { Trastuzumab-treated: } 22.3 \text { weeks; trastuzumab- } \\
\text { naive: } 39.6 \text { weeks }\end{array}$ \\
\hline & 2008 & NCT00777101 & $\begin{array}{l}\text { HER2-positive, locally advanced or metastatic breast cancer patients; progression } \\
\text { on or following 1-2 prior trastuzumab regimens; and prior taxane treatment in the } \\
\text { neoadjuvant, adjijuant, locally advanced and/or metastatic disease treatment settings }\end{array}$ & Randomized, open-label, phase II & Neratinib $(n=117)$, lapatinib + capecitabine $(n=116)$ & $29 \%, 41 \% ; P=0.067$ & 4.5 months, 6.8 months; $\mathrm{HR}=1.19 ; \mathrm{P}=0.231$ \\
\hline T-DM1 & 2009 & EMLLA study & $\begin{array}{l}\text { HER2-positive, unresectable, locally advanced or metastatic breast cancer who were } \\
\text { previously treated with trastuzumab and a taxane }\end{array}$ & Randomized, open-label, phase 3 trial & T-DM1 (n=495), lapatinib plus capecitabine (n=496) & $43.6 \%, 30.8 \% ; P<0.001$ & 9.6 months, 6.4 months; $\mathrm{HR}=0.65 ; \mathrm{P}<0.001$ \\
\hline Lapatinib + capecitabine & 2004 & EGF100151 study & $\begin{array}{l}\text { HER2-positive, locally advanced or metastatic breast cancer that had progressed after } \\
\text { treatment with regimens that included an anthracycline, a taxane, and trastuzumab }\end{array}$ & Phase 3, randomized, open-label study & Lapatinib + capecitabine $(n=163)$, capecitabine alone $(n=161)$ & $22 \%, 14 \%$ & $\begin{array}{l}\text { Time to progression: } 8.4 \text { months, } 4.4 \text { months; } \\
H R=0.51 ; \text { P }<0.001\end{array}$ \\
\hline Neratinib + capecitabine & 2014 & NCT00741260 & 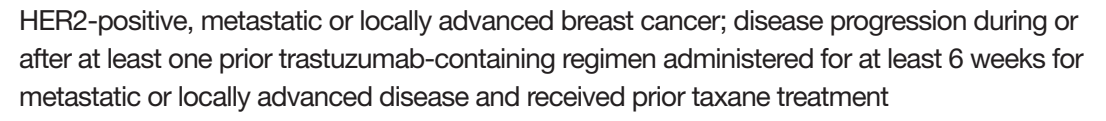 & Phase 2 & $\begin{array}{l}\text { Neratinib plus capecitabine: no prior lapatinib ( } n=61) \text {, prior } \\
\text { lapatinib }(n=7)\end{array}$ & $64 \%, 57 \%$ & 40.3 weeks, 35.9 weeks \\
\hline
\end{tabular}

HER2, human epidermal growth factor receptor 2; TKI, tyrosine kinase inhibitior; ORR, objective response rate; PFS, progression-free survival; HR, hazard ratio. 